\title{
OPTIMAL ELECTRODE POSITION FOR DELAMINATION DETECTION IN CARBON FIBRE REINFORCED POLYMER COMPOSITES USING ELECTRIC RESISTANCE CHANGE METHOD
}

\author{
Andrejs Kovalovs, Andris Chate \\ Riga Technical University, Latvia \\ andrejs.kovalovs@rtu.lv
}

\begin{abstract}
The electric resistance measure by the four probe method is used by various researchers for detection of the internal delamination in laminated carbon-fiber-reinforced polymers composite material. In the present study, the effect of spacing between probes on the crack detection efficiency has been investigated. The problem has been solved by the method of planning of the experiment and the response surface method by using the 2-D finite element model. The distance between the probes and the crack length has been selected as the parameter of the study. As a result, it was revealed, that in case of the crack growth, a decrease in the distance between the current and voltage probes has a greater effect on the detection of the crack than the change in the distance between only the current probes.
\end{abstract}

Keywords: CFRP, delamination, electrical resistance, non-destructive, ANSYS.

\section{Introduction}

Modern composite materials, such as carbon-fiber-reinforced polymers (CFRP), have unique physical and mechanical properties. Their lightness, high strength, durability, reliability, longevity, and different other characteristics ensure extensive engineering applications in various industries. However, composite materials also have a number of disadvantages. External mechanical loads, repetitive cyclic stresses and impulsive loads lead to the appearance of various defects, such as rupture of fibers or delamination in the material. These damages are invisible on the surface of composite elements, which can lead to a serious weakening of the structure and tragic consequences. Therefore, the non-destructive inspection for the identification of the cracks and delamination of composite elements is required.

Currently, there are various methods of non-destructive inspection, which make it possible to monitor the technical condition of composite elements. These methods include optical methods (thermography, shearography), acoustic emission, computer tomography, traditional ultrasonic testing, ultrasonic examination and the electrical resistance method [1].

The electric resistance method is used by various researchers to detect the internal delamination in composite materials, since this method does not require expensive tools, and is applicable to various structures. The main idea of this method implies that the damage, for example, fiber breakage or delamination between layers in CFRP composite material, leads to a decrease in electrical conductivity in the damaged area, and results in a change in electrical voltage.

Many publications that demonstrate the use and applicability of this method for the assessment of the damage in laminated fiber composites have appeared over the past decades. A brief review of the literature can be found in the paper [2].

Most of the papers in this area were created by Todoroki and his co-authors (Todokori et al.) [310]. Todoroki used the method of electrical resistance to determine the sizes and location of delamination in CFRP laminated plate by using multiband electrodes located on the specimen surface. By using the finite-element modeling, the distribution of the electric field and the current inside the specimen was investigated. These calculations were made to understand the orthotropic electrical properties of the unidirectional CFRP [4] and were further expanded to a multi-layer composite with different laminating [5]. The delamination in the specimens was created on laboratory equipment by using the three-point bending scheme. The size of the damage and the location were checked by ultrascaning. Electrical resistance was measured before and after the experiment. The two-probe and four-probe methods were used for the study [8]. In parallel, the numerical studies were performed to assess the damage and its location. By using the method of finite-element modeling, the results of damage identification by means of the surface response method were presented in the research papers $[5 ; 10]$. It was concluded that the use of surface response is very effective for identification of delamination in CFRP. 
The study of electrical resistance continues to our days. In the papers [11-13], the authors conduct experiments to determine the electrical resistance of a multilayer composite. The results of the experiment are numerically and analytically compared.

The objective of this paper is to study the relationship between the change in the electrical potential of the unidirectional CFRP specimen and the distance between the probes in order effectively to detect the crack. A similar problem was explored in the paper [9]. The problem was solved by finite-element modeling of 5 specimens with equally changing the distance between seven electrodes. The results showed that the smallest distance between the electrodes in the CFRP composite provides the best result for the detection of cracks and delamination in the composite. The difference of this work lies in the fact that the distance between the internal and external probes does not change regularly when using the four-probe method. The problem is solved by the method of experiment planning and response surface method (RSM) with the use of 2-D finite element model. Electric resistance percentage change between a damaged and undamaged composite specimen is considered as an approximation function.

\section{Materials and methods}

Carbon fiber-reinforced composite materials are electrically conductive due to the high conductivity along the carbon fiber. Despite the fact that the polymer matrix in the composite material acts as an insulator, the contact between the fibers creates electrical conductivity, which allows the current to flow both in the transverse direction and through-the-thickness. In this case, the electrical conductivity in cross direction and through-the-thickness is much lower than along the fibers. The electrical resistance of a composite specimen depends on the fiber volume fraction of CFRP composite material, fiber size and the laminate sequence [1].

The method of measurement of electrical resistance is based on transmission of a direct current through the specimen and determination of the drop in voltage between the contacts. The electrical resistance $\mathrm{R}$ is calculated according to Ohm's law: $R=V / I$, where $V$ is the voltage between the electrodes, $I$ is the current intensity.

Four-probe methods are used to measure the electric resistance of a composite specimen and detection of delamination in the composite material. This method is based on determination of the electrical resistance, when the electric current is passed through the external probes, and the voltage is measured between the internal probes. Based on the measured voltage and current, the resistance between the voltage contacts is measured.

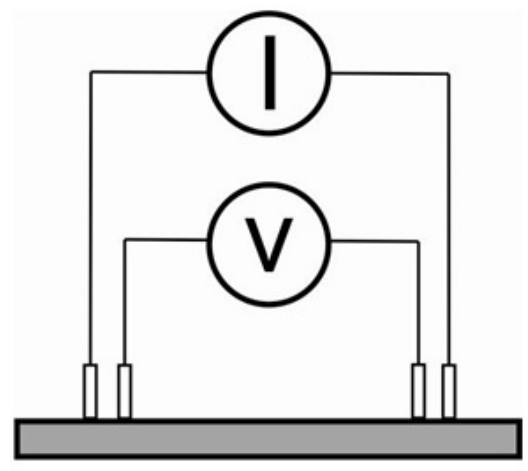

Fig. 1. Four probe method [2]

For the numerical calculation of the spacing effect between the probes on the electrical resistance we will explore the unidirectional carbon-fiber composite in plane in Fig. 2. The specimen length is $200 \mathrm{~mm}$ and the thickness is $2 \mathrm{~mm}$. The height of the probe is $0.5 \mathrm{~mm}$ and the width is $2 \mathrm{~mm}$. 4 probes are placed on the surface of the specimen when measuring the surface resistance.

The electrical conductivity along the fiber is taken up as $\sigma_{0}=5500 \mathrm{~S} \cdot \mathrm{m}^{-1}$. The electrical conductivity in thickness is taken up as $\sigma_{t}=20.9 \mathrm{~S} \cdot \mathrm{m}^{-1}$. The data correspond to fiber volume fractions of $62 \%$ [4]. The material of the probes is silver, the conductivity of which is equal to $62.9 \mathrm{~S} \cdot \mathrm{m}^{-1}$.

The calculation was performed by using the commercial finite-element software ANSYS 16.0. The two-dimensional finite-element model of the composite specimen was developed for the study, 
which was constructed with the use of the element PLANE230. The size of the element was chosen based on the study of the mesh convergence. The crack in the specimen is created by cutting a number of elements from the finite-element model. The crack thickness is $0.1 \mathrm{~mm}$ and is located at the same distance between the upper and lower surfaces of the specimen.

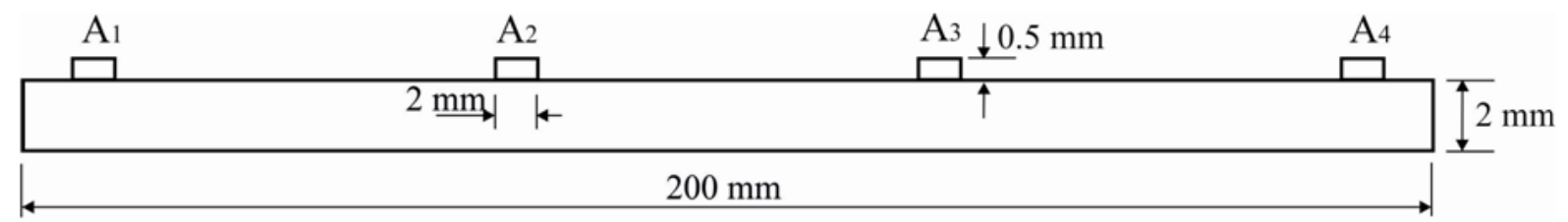

Fig. 2. Cross-section dimensions of composite specimen

To calculate the surface resistance $R$ by the four-probe method, the electric current $I$ of $30 \mathrm{~mA}$ is transmitted from the external probe $A_{1}$ to the probe $A_{4}$. The electric voltage $V$ at the probe $A_{2}$ is assumed to be $0 \mathrm{~V}$. The resulting difference of electric potentials at the probe $A_{3}$ is divided by the transmitted electric current of $30 \mathrm{~mA}$ for the calculation of the surface resistance between the internal probes $\mathrm{A}_{2} \mathrm{~A}_{3}$. The calculation is made for the specimens with damage and without damage. By using the ratio of the obtained results, the Electric Resistance Percentage Change $R \%$ is calculated as:

$$
R_{\%}=\left(1-\frac{R_{0}}{R_{d}}\right) \cdot 100 \%,
$$

where $R_{d}$ - electrical resistance of a specimen with delamination, V;

$R_{0}$ - electrical resistance of a specimen without delamination, $\mathrm{V}$.

The purpose of this study is the identification of the functional relationship between the distance of the probes and the percentage change in electrical resistance. The solution of the problem is divided into several stages: choice of the design parameters and the interval of variation of each parameter, construction of the experiment plan for the selected parameters, execution of the numerical experiment and determination of the general form of the regression equation according to the results of the numerical experiments.

The plan of the experiment is formulated for 3 design parameters (Fig. 3): the distance between the internal probes that are measuring the specimen resistance $\left(l_{1}\right)$, the distance between the internal and external probes $\left(l_{2}\right)$ and the crack length $\left(l_{\text {crack }}\right)$. The 3D plan of the experiment is shown in Fig. 4. Thus 48 numerical experiments were obtained that should be calculated to construct the approximating function.

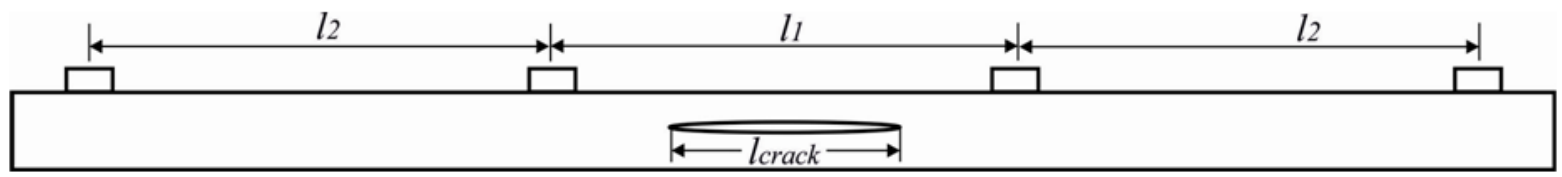

Fig. 3. Design parameters

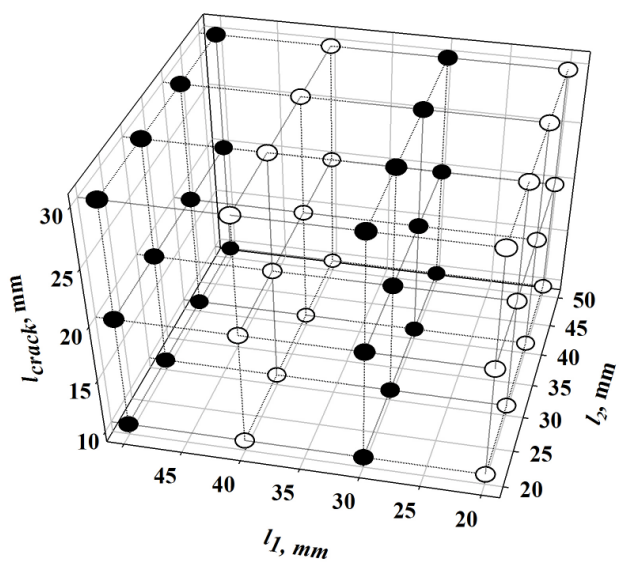

Fig. 4. Plan of experiment: 3D-View 
Using the points of the experiment plan, the finite-element solutions were obtained, the numerical results of which were used to determine the Electric Resistance Percentage Change and to construct the approximating function.

\section{Results and discussion}

The numerical data obtained by the finite element calculations in the points of the plan of experiments has been used to build the approximating functions by using the program RESINT [14]. A polynomial equation is given as follow:

$$
\begin{aligned}
& \mathrm{Y}=2.46-6.34 \cdot 10^{-3} \cdot l_{2} \cdot l_{\text {crack }}-6.89 \cdot 10^{-3} \cdot l_{1} \cdot l_{2}+1.23 \cdot 10^{-2} \cdot l_{\text {crack }}^{2}+2.95 \cdot 10^{-4} \cdot l_{1} \cdot l_{2} \cdot l_{\text {crack }}+ \\
& +1.44 \cdot 10^{-5} \cdot l_{2}^{3}+1.41 \cdot 10^{-4} \cdot l_{1} \cdot l_{\text {crack }}+2.39 \cdot 10^{-4} \cdot l_{1} \cdot l_{\text {crack }}^{2}-1.18 \cdot 10^{-4} \cdot l_{2} \cdot l_{\text {crack }}^{2}-7.40 \cdot 10^{-5} \cdot l_{1} \cdot l_{2}
\end{aligned},
$$

Response surface is verified by the finite element solutions in the points different from the points taken in the plan of experiments. Examples of finite element verification with the response surfaces of unidirectional CFRP composites are presented in Fig. 5. A good correlation is observed for the approximations and finite element solutions.
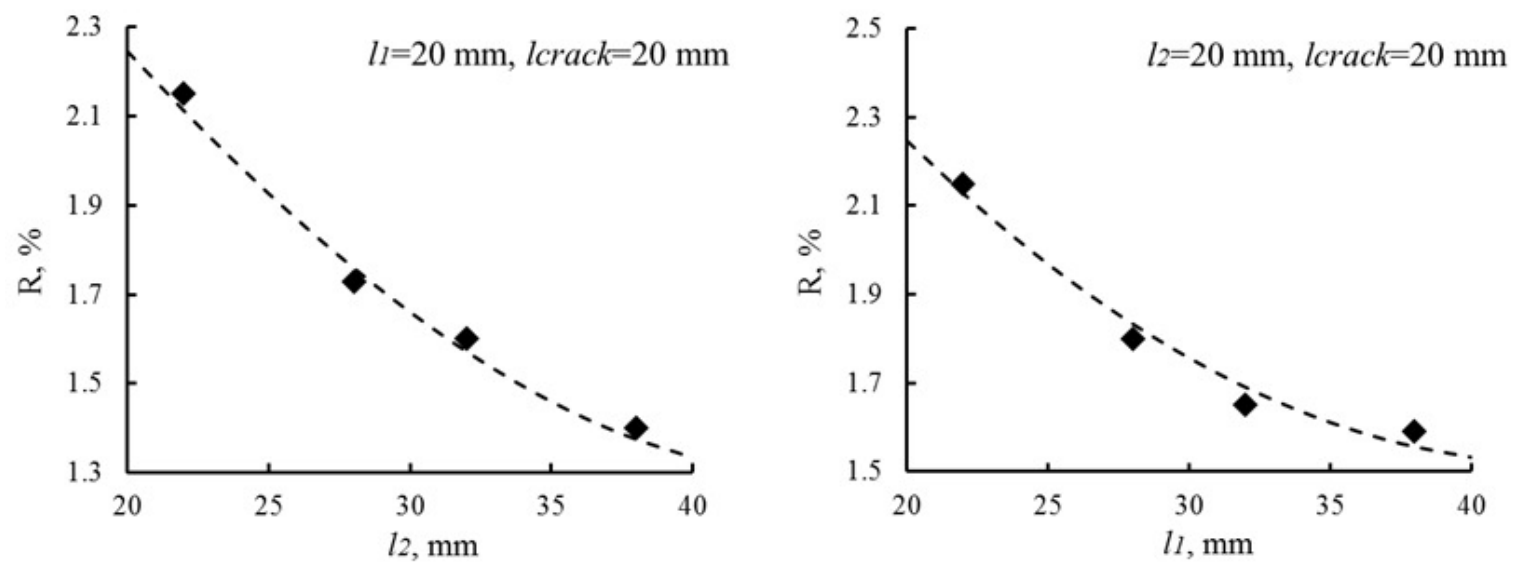

Fig 5. Accordance between approximation function and control points

Upon selection of the equation of regression the parametric studies are carried out to study the influence of the design parameters on the change in electrical resistance percentage. Fig. 6-7 show the response surface plot of the effect of distances between probes on the electrical resistance change.

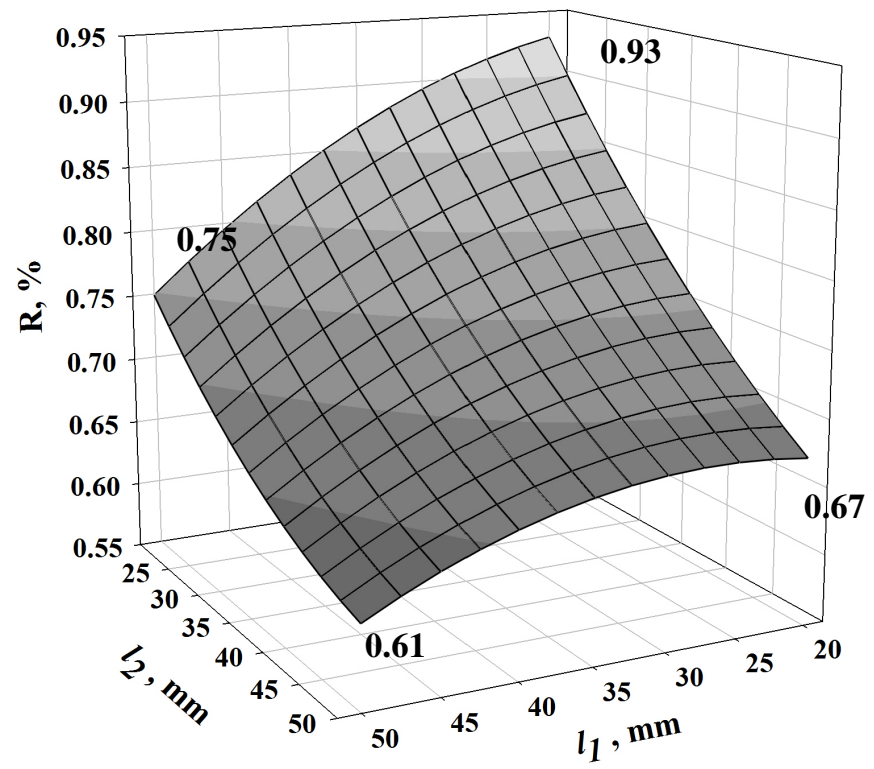

Fig. 6. Dependency on electrical resistance change: $l_{\text {crack }}=10 \mathrm{~mm}$ 
Fig. 6 shows the dependency of the change in the electrical resistance percentage on the distance between the internal probes that are measuring the specimen resistance $\left(l_{1}\right)$ and the distance between the internal and external probes $\left(l_{2}\right)$. The length of the crack is $10 \mathrm{~mm}$ at least.

It can be seen that, when the distance between the internal and external probes $\left(l_{2}\right)$ is minimal, the resistance percentage value grows significantly. When the distance between the internal probes $\left(l_{1}\right)$ grows, the value of the resistance percentage significantly increases with a decrease of the distance between the internal and external probes $\left(l_{2}\right)$. Maximal value of resistance percentage achieves $0.93 \%$, when the distance between the probes $\left(l_{1}, l_{2}\right)$ is minimal and is equal to $20 \mathrm{~mm}$.

The dependence of the distances between the probes on the resistance percentage, when the length of the crack is maximum, is illustrated in Fig 6. In this case the resistance percentage begins to grow with a decrease of the distances between all probes $\left(l_{1}, l_{2}\right)$. The maximum resistance percentage reaches $2.28 \%$ for minimal distances between the probes, respectively. It can be observed that the smallest values of the percentage changes correspond to the greater distance of probes.

Fig. 7 shows that the reduction of the distance between the internal and external probes $\left(l_{2}\right)$ makes it possible more effectively to detect the crack while growing. It can be seen that in case of the minimum distance between the internal and external probes $\left(l_{2}\right)$ the resistance percentage increases from $1.94 \%$ to $2.28 \%$. The values of resistance percentage are changing by 1.17 times. In case of the maximum distance between the internal and external probes $\left(l_{2}\right)$ the resistance percentage increases from $1.48 \%$ to $1.57 \%$. The values of resistance percentage are changing by 1.05 times. On the other hand, if the distance between the internal probes $\left(l_{1}\right)$ is maximum, then the resistance percentage value increases from $1.48 \%$ to $1.94 \%$. The values of resistance percentage are changing by 1.3 times. In case of a minimum distance between the internal probes $\left(l_{1}\right)$ the resistance percentage increases from $1.57 \%$ to $2.28 \%$. The values of resistance percentage are changing by 1.45 times. Thus, it can be seen that in case of the crack growth a decrease in the distance between the internal and external probes has a greater effect on the detection of the crack than the change in the distance between the internal probes.

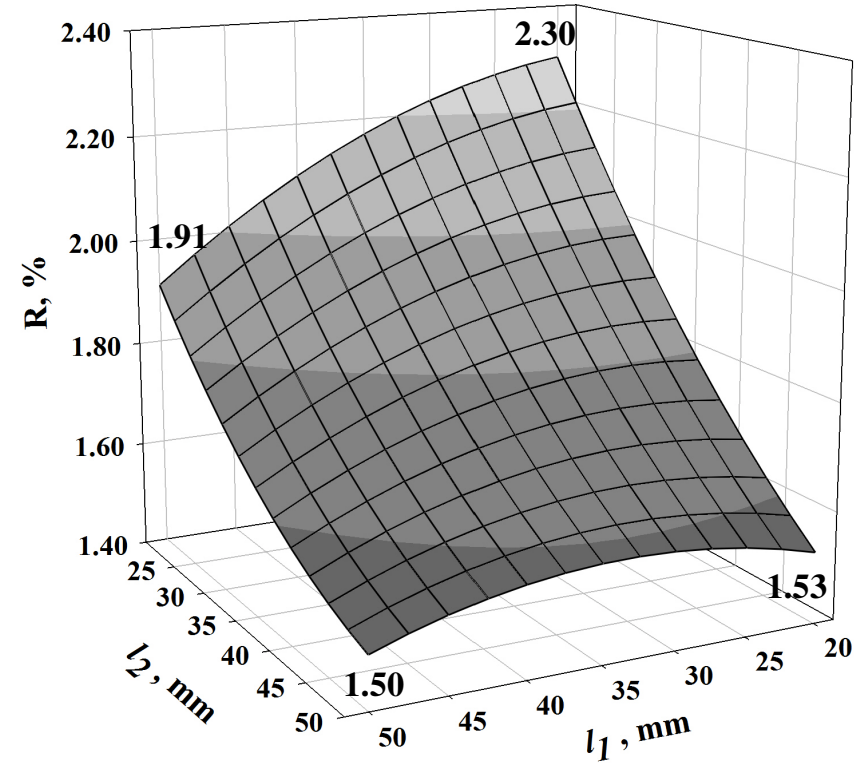

Fig. 7. Dependency on electrical resistance change: $l_{\text {crack }}=30 \mathrm{~mm}$

\section{Acknowledgments}

This work was supported by the Latvian Ministry of Education and Science (RTU grant No. 7045.5).

\section{Conclusions}

1. A 2-D numerical analysis of the electrical resistance for strip-type composite specimens with and without delamination is carried out. The surface resistances are numerically calculated using the 
four-probe method. The planning of experiments and the response surface techniques were used to obtain the optimal distance between probes to achieve the maximal electric resistance percentage change.

2. The present study shows that the electric resistance percentage change is dependent on the location of the probe pairs used for the resistance measurement. The maximal value of resistance percentage is achieved $2.28 \%$, when the distance between all probes is minimal $-20 \mathrm{~mm}$ and the length of the crack is maximal $30 \mathrm{~mm}$.

3. Analysis of the dependency on the electrical resistance change shows that in case of the crack growth, a decrease in the distance between the internal and external probes has a greater effect on the detection of the crack than the change in the distance between the internal probes.

\section{References}

[1] Gholizadeh S. A review of non-destructive testing methods of composite materials. Procedia Structural Integrity, Vol. 1, 2016, pp. 50-57.

[2] Wen J., Xia Z., and Choy E. F. Damage detection of carbon fiber reinforced polymer composites via electrical resistance measurement. Composites Part B: Engineering, Vol. 42(1), 2011, pp. 7786.

[3] Todoroki A. The effect of number of electrodes and diagnostic tool for monitoring the delamination of CFRP laminates by changes in electrical resistance. Composites Science and Technology, Vol. 61(13), 2001, pp. 1871-1880.

[4] Todoroki A., Tanaka M., and Shimamura Y. Measurement of orthotropic electrical conductance of CFRP laminates and analysis of the effect on delamination monitoring with electrical resistance change method. Composites Science and Technology, Vol. 62(5), 2002, pp. 619-628.

[5] Todoroki A., Tanaka Y. Delamination identification of cross-ply graphite/epoxy composite beams using electric resistance change method. Composites Science and Technology, Vol. 62(5), 2002, pp. 629-639.

[6] Todoroki A., Tanaka M., and Shimamura Y. Delamination monitoring of graphite/epoxy laminated composite plate of electrical resistance change method. Composites Science and Technology, Vol. 62(9), 2002, pp. 1151-1160.

[7] Todoroki A., Tanaka M., and Shimamura Y. High performance estimations of delamination of graphite/epoxy laminates with electric resistance change method. Composites Science and Technology, Vol. 63(13), 2003, pp. 1911-1920.

[8] Todoroki A., Tanaka M., and Shimamura Y. Multi-probe electric potential change method for delamination monitoring of graphite/epoxy composite plates using normalized response surface. Composites Science and Technology, Vol. 64(5), 2004, pp. 749-758.

[9] Todoroki A., Tanaka M., and Shimamura Y. Electrical Resistance Change Method for Monitoring Delaminations of CFRP Laminates: Effect of Spacing between Electrodes. Composites Science and Technology, Vol. 65(1), 2005, pp. 37-46.

[10] Iwasaki A., Todoroki A. Statistical Evaluation of Modified Electrical Resistance Change Method for Delamination Monitoring of CFRP Plate. Structural Health Monitoring, Vol. 4(2), 2005, pp. 119-136.

[11]Zappalorto M., Panozzo F., Carraro P.A., Quaresimin M. Electrical response of a laminate with a delamination: modelling and experiments. Composites Science and Technology, Vol. 143, 2017, pp. 31-45.

[12]Panozzo F., Zappalorto M., Maragonia L., Nothdurfter S.K., Rullo A., and Quaresimin M. Modelling the electrical resistance change in a multidirectional laminate with a delamination. Composites Science and Technology, Vol. 162, 2018, pp. 225-234.

[13] Panozzo F., Zappalorto M., Carraro P.A., Quaresimin M. Electrical resistance change vs damage state in cracked symmetric laminates: a closed for solution. Composites Science and Technology, Vol. 184, 2018, pp. 1081-1091.

[14]Eglajs V. Approximation of Data by the Multi-dimensional Equation of Regression. Problems of Dynamics and Strength, Vol. 39, 1981, pp. 120-125. 